\title{
Online quality assessment of human movement from skeleton data
}

\section{Adeline Paiement}

csatmp@bristol.ac.uk

Lili Tao

lili.tao@bristol.ac.uk

Sion Hannuna

sh1670@bristol.ac.uk

Massimo Camplani

massimo.camplani@bristol.ac.uk

Dima Damen

dima.damen@bristol.ac.uk

Majid Mirmehdi

majid@cs.bris.ac.uk
Visual Information Laboratory

Department of Computer Science

University of Bristol

Bristol, UK
This work addresses the challenge of analysing the quality of human movements from visual information which has use in a broad range of applications, from diagnosis and rehabilitation to movement optimisation in sports science. Traditionally, such assessment is performed as a binary classification between normal and abnormal by comparison against normal and abnormal movement models, e.g. [5]. Since a single model of abnormal movement cannot encompass the variety of abnormalities, another class of methods only compares against one model of normal movement, e.g. [4]. We adopt this latter strategy and propose a continuous assessment of movement quality, rather than a binary classification, by quantifying the deviation from a normal model. In addition, while most methods can only analyse a movement after its completion e.g. [6], this assessment is performed on a frame-by-frame basis in order to allow fast system response in case of an emergency, such as a fall.

Methods such as $[4,6]$ are specific to one type of movement, mostly due to the features used. In this work, we aim to represent a large variety of movements by exploiting full body information. We use a depth camera and a skeleton tracker [3] to obtain the position of the main joints of the body, as seen in Fig. 1. We normalise this skeleton for global position and orientation of the camera, and for the varying height of the subjects, e.g. using Procrustes analysis.

The normalised skeletons have high dimensionality and tend to contain outliers. Thus, the dimensionality is reduced using Diffusion Maps [1] which is modified by including the extension that Gerber et al. [2] presented to deal with outliers in Laplacian Eigenmaps. The resulting high level feature vector $\mathbf{Y}$, obtained from the normalised skeleton at one frame, represents an individual pose and is used to build a statistical model of normal movement.

Our statistical model is made up of two components that describe the normal poses and the normal dynamics of the movement. The pose model is in the form of the probability density function (pdf) $f_{Y}(y)$ of a random variable $Y$ that takes as value $y=\mathbf{Y}$ our pose feature vector $\mathbf{Y}$. The pdf is learnt from all the frames of training sequences that contain normal instances of the movement, using a Parzen window estimator. The quality of a new pose $y_{t}$ at frame $t$ is then assessed as the log-likelihood of being described by the pose model, i.e.

$$
l l h_{\text {pose }}=\log f_{Y}\left(y_{t}\right) .
$$

The dynamics model is represented as the pdf $f_{Y_{t}}\left(y_{t} \mid y_{1}, \ldots, y_{t-1}\right)$ which describes the likelihood of a pose $y_{t}$ at a new frame $t$ given the poses at the previous frames. In order to compute it, we introduce $X_{t}$ with value $x_{t} \in$ $[0,1]$, which is the stage of the (periodic or non-periodic) movement at frame $t$. Note, in the case of periodic movements, this movement stage can also be seen as the phase of the movement's cycle. Based on Markovian assumptions, we find that

$$
f_{Y_{t}}\left(y_{t} \mid y_{1}, \ldots, y_{t-1}\right) \approx f_{Y_{t}}\left(y_{t} \mid \hat{x}_{t}\right) f_{X_{t}}\left(\hat{x}_{t} \mid \hat{x}_{t-1}\right),
$$

with $\hat{x}_{t}$ an approximation of $x_{t}$ that minimises $f_{\left\{X_{0}, \ldots, X_{t}\right\}}\left(x_{0}, \ldots, x_{t} \mid y_{1}, \ldots, y_{t}\right)$ [ $f_{Y_{t}}\left(y_{t} \mid x_{t}\right)$ is learnt from training sequences using Parzen window estimation, while $f_{X_{t}}\left(x_{t} \mid x_{t-1}\right)$ is set analytically so that $x_{t}$ evolves steadily during a movement. The dynamics quality is then assessed as the loglikelihood of the model describing a sequence of poses within a window
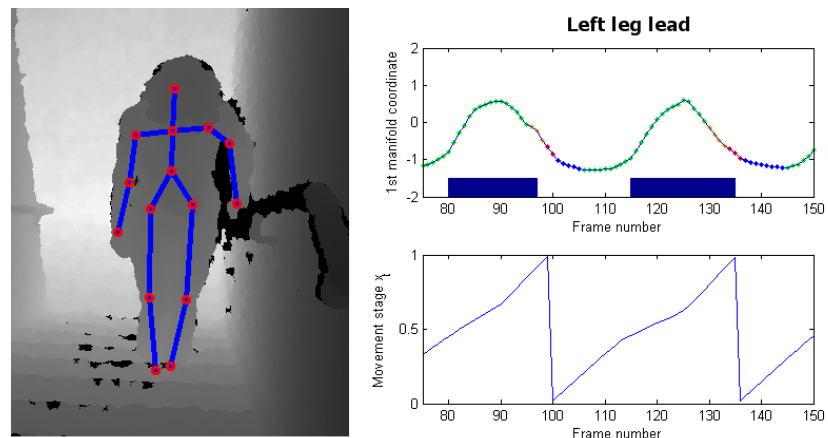

This work was performed under the SPHERE IRC funded by the UK Engineering and Physical Sciences Research Council (EPSRC), Grant EP/K031910/1.

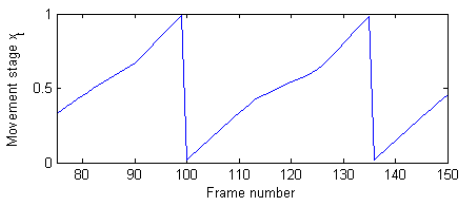

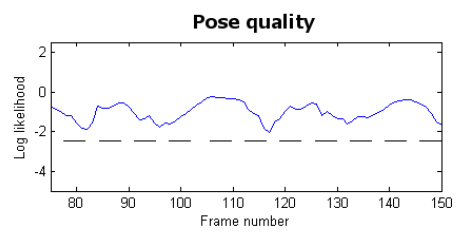

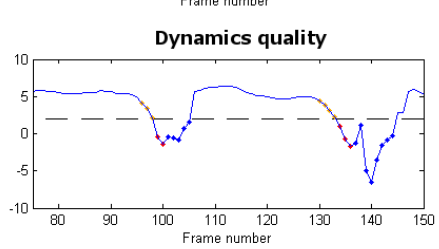

Figure 1: Analysis of gait on stairs. Clockwise from top left: raw skeleton data, high level description of the gait, dynamics and pose quality measures.

of size $\omega$ :

$$
l l h_{s e q} \approx \frac{1}{\omega} \sum_{i=t-\omega+1}^{t} \log \left(f_{Y_{i}}\left(y_{i} \mid x_{i}\right) f_{X_{i}}\left(x_{i} \mid x_{i-1}\right)\right) .
$$

In our experiments, these two quality measures provided a continuous quality assessment of gait on stairs on a frame-by-frame basis, as illustrated at the bottom of Fig. 1. Two thresholds, set empirically, allowed deciding when gait becomes abnormal. More details and experiments can be found in the article and on our project's webpage ${ }^{1}$.

[1] R. R. Coifman and S. Lafon. Diffusion maps. Applied and computational harmonic analysis, 21(1):5-30, 2006.

[2] S. Gerber, T. Tasdizen, and R. Whitaker. Robust non-linear dimensionality reduction using successive 1-dimensional Laplacian eigenmaps. In Proc. of Int. Conf. on Machine Learning, pages 281-288. ACM, 2007.

[3] OpenNI User Guide. OpenNI organization, November 2010. URL http://www.openni.org/documentation.

[4] J. Snoek, J. Hoey, L. Stewart, R. S. Zemel, and A. Mihailidis. Automated detection of unusual events on stairs. Image and Vision Computing, 27(1):153-166, 2009.

[5] M. Z. Uddin, J. T. Kim, and T. S. Kim. Depth video-based gait recognition for smart home using local directional pattern features and hidden Markov model. Indoor and Built Environment, 23(1):133-140, 2014.

[6] R. Wang, G. Medioni, C. J. Winstein, and C. Blanco. Home monitoring musculo-skeletal disorders with a single 3D sensor. In $\underline{\mathrm{CVPR}}$ Workshops, pages 521-528. IEEE, 2013.

\footnotetext{
${ }^{1}$ www.irc-sphere.ac.uk/work-package-2/movement-quality
} 